\title{
Modelling the Effect of Genes on the Dynamics of Probabilistic Spiking Neural Networks for Computational Neurogenetic Modelling
}

\author{
Nikola Kasabov $^{1,2}$, Stefan Schliebs ${ }^{1}$, Ammar Mohemmed $^{1}$ \\ ${ }^{1}$ Knowledge Engineering and Discovery Research Institute \\ Auckland University of Technology, Auckland 1010, New Zealand \\ \{nkasabov, sschlieb,amohemme\}@aut.ac.nz \\ ${ }^{2}$ Institute for Neuroinformatics, University of Zurich and ETH
}

Keywords: Computational neurogenetic modeling; Spiking neural networks; Gene regulatory networks; Probabilistic neural models.

\begin{abstract}
Computational neuro-genetic models (CNGM) combine two dynamic models - a gene regulatory network (GRN) model at a lower level, and a spiking neural network (SNN) model at a higher level to model the dynamic interaction between genes and spiking patterns of activity under certain conditions. The paper demonstrates that it is possible to model and trace over time the effect of a gene on the total spiking behavior of the SNN when the gene controls a parameter of a stochastic spiking neuron model used to build the SNN. Such CNGM can be potentially used to study neurodegenerative diseases or develop CNGM for cognitive robotics.
\end{abstract}

\section{Introduction}

Computational Neuro Genetic Modelling (CNGM) is a biologically motivated modeling approach that is concerned with the creation of two-level hierarchical computational models, where interaction between large number of dynamic variables (called genes) is modeled over time as a gene-regulatory network model (GRN) that affects the activity of a higher level system - modeled as a spiking neural network (SNN). The behavior of the two systems, in their continuous interaction under certain inputoutput conditions, have been introduced and studied in [Kasabov and Benuskova, 2004, Kasabov and Benuskova, 2005, Kasabov et al., 2005, Kasabov, 2007, Kasabov, 2009, Kasabov, 2008, Kasabov et al., 2011]. CNGM constitute the next generation of computational modeling techniques built on the foundations of the traditional neural network techniques.

The goal of this paper is to explore and to develop further the CNGM paradigm through the introduction of stochastic neuronal models (e.g. [Gerstner and Kistler, 2002]) used to build stochastic/probabilistic SNN (pSNN). Such pSNN are more biologically plausible, offering some additional advantages [Kasabov et al., 2011]. For this purpose genes are used to control parameters and their effect on the behavior of the whole pSNN is modeled and studied.

A specific gene from the genome relates to the activity of a neuronal cell by means of a specific protein. Complex interactions between genes and proteins within the internal gene/protein regulatory network influence the functioning of each neuron and a neural network as a whole [Marcus, 2005, Holter et al., 2001]. With the advancement of molecular research technologies huge amount of data and information is available about the genetic basis of neuronal functions and diseases [Marcus, 2005, Holter et al., 2001, NCBI, 2003]. This information can be utilized to create models of brain functions and diseases that include models of gene interactions within models of neural networks.

In order to create biologically plausible CNGM we need to integrate knowledge from genomics, proteomics, neuroscience, psychology, and theoretical disciplines such as 


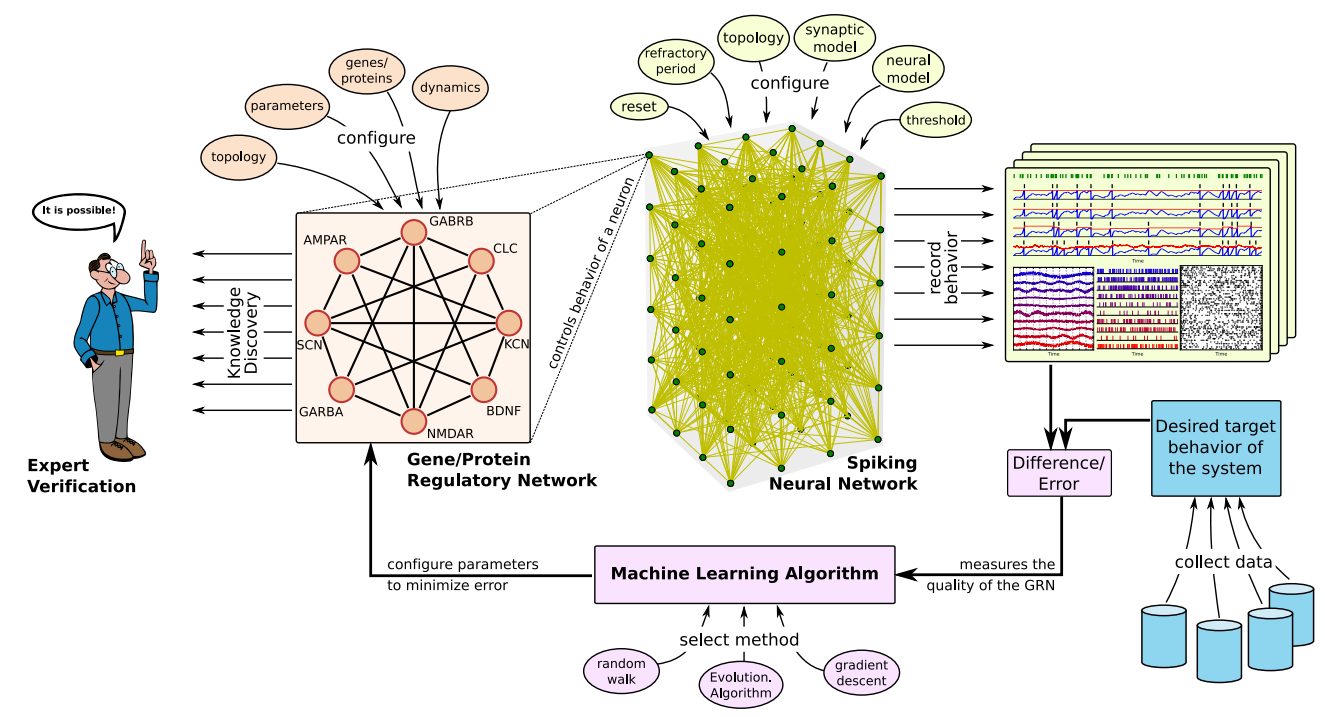

Figure 1: A schematic illustration of the Computational Neurogenetic Modelling framework.

computer science and physics. To understand the functions of genes, we need to know how they are expressed, when they are expressed, conservation among their products and their response to therapeutic drugs. In addition, we also need to know how they interact and influence the dynamics of neurons in neural networks.

Genetic neuroscience uses principles from cellular and molecular biology to investigate questions about gene actions in the brain. Many techniques are designed to collect data related to the effect of genes and proteins on brain functions, e.g. genetic mutations; gene knockouts; protein purification; gene expression analysis; GAS chromatography; gel electrophoresis; high performance liquid chromatography (HPLC); western blotting, etc. Another multi-gene approach, high throughput genotyping technology, can be used to define single nucleotide polymorphisms that characterize diseases. All these useful techniques however need to be complemented and combined with sophisticated bioinformatics techniques in order to discover complex gene/protein interactions in relation to a given objective function. This is where CNGM can help.

The functioning of a CNGM is illustrated schematically in Figure 1. A Gene/Protein Regulatory Network (GRN) models the interaction of genes/proteins over time. Each of these genes/proteins can affect the behavior of a connected spiking neuron by directly controlling its parameters. For example, some proteins affect the excitability of the neuron (e.g. AMPAR, NMDAR), while others promote neural inhibition (e.g. GARBA, GARBRB) [Kasabov and Benuskova, 2004, Kasabov and Benuskova, 2005, Kasabov et al., 2005, Kasabov, 2007, Kasabov, 2009, Kasabov, 2008, Kasabov et al., 2011]. By changing the dynamics of the GRN, a specific behavior can be imposed to the SNN. An optimization algorithm can be used to modify the parameters of the GRN in such a way that a desired SNN output characteristic is obtained. This behavior could, for example, resemble real-world data obtained from clinical experiments. The optimized GRN that causes the desired behavior of the SNN is now expected to contain valuable information about the relationship and dynamics of the involved genes. Studying these dynamics may provide interesting new insights in the area of genetic neuroscience and facilitate new discoveries Several initial studies have investigated the feasibility of the CNGM approach. For example, in [Villa et al., 2005], a protein regulatory network (PRN) model was obtained from induced epileptic seizure EEG data from mice. With the use of a simple neural model (Integrate-and-Fire [Abbott, 1999]), a genetic algorithm [Fogel, 2006] was used to optimized the PRN model to match some real data [Benuskova and Kasabov, 2007].

Using a CNGM approach to build robots was investigated in [Meng et al., 2010]. 
The functions of the robot are distributed between a SNN model and a PRN model that enables to implement a principally new concept of functional and structural flexibility in robots. Robotic systems that employed PRN were also introduced as epigenetic robots [Morse et al., 2010].

The studies in CNGM so far have used simple and deterministic spiking neuron models, such as the Leaky Integrate-and-Fire (LIF) model [Abbott, 1999]. However it has already been demonstrated that using probabilistic/stochastic spiking neuron models, that are more biologically plausible, enhances the functionality and the applicability of SNN. The probabilistic approach is motivated by the fact that biological neurons exhibit significant stochastic characteristics. Including non-deterministic elements into the neural model would bring benefits for modelling brain information processes and for modelling stochastic engineering processes too.

One of the problems of using pSNN is to optimize and control the neuronal parameters, even though for many biologically plausible parameters it is already known what genes/protein control them. In a simple experiment this paper demonstrates that it is possible to model the effect of a gene, that controls a particular neuronal parameter, on the spiking activity of the whole pSNN. This will enable a further study and development of pCNGM and their applications.

\section{Probabilistic Neural Models}

In this section, we describe the probabilistic neural models that we have used to replace the deterministic LIF neurons of a traditional SNN. In this study, we employ some very simple probabilistic extensions of the LIF model.

Models of probabilistic neurons have been proposed in several studies, e.g. in the form of dynamic synapses [Maass and Zador, 1999], the stochastic integration of the post-synaptic potential [Gerstner and Kistler, 2002] and stochastic firing thresholds [Clopath et al., 2007].

In [Kasabov, 2010] a probabilistic neuronal model is introduced that has three probabilistic parameters to extend the LIF model: $p_{c j, i}(t)$ is the probability that a spike emitted by neuron $n_{j}$ will reach neuron $n_{i}$ at a time moment $t$ trough the connection between $n_{j}$ and $n_{i} ; p_{s j, i}(t)$ is the probability of the synapse $s_{j, i}$ to contribute to the post synaptic potential $P S P_{i}(t)$ after the latter has received a spike from neuron $n_{j} ; p_{i}(t)$ is the probability parameter for the neuron $n_{i}$ to emit an output spike at time $t$, once the total post-synaptic potential $P S P_{i}(t)$ has reached a value above the PSP threshold (a noisy threshold). As a partial case, when all or some of the probability parameters are fixed to " 1 ", the pSNM can be reduced to the LIF.

The LIF neuron is arguably the best known model for simulating spiking networks. It is based on the idea of an electrical circuit containing a capacitor with capacitance $C$ and a resistor with resistance $R$, where both $C$ and $R$ are assumed to be constant. The model dynamics are then described by the following differential equation:

$$
\tau_{m} \frac{d u}{d t}=-u(t)+R I(t)
$$

The constant $\tau_{m}$ is called the membrane time constant of the neuron. Whenever the membrane potential $u$ crosses a threshold $\vartheta$ from below, the neuron fires a spike and its potential is reset to a resting potential $u_{r}$. It is noteworthy that the shape of the spike itself is not explicitly described in the traditional LIF model. Only the firing times are considered to be relevant.

We define a stochastic reset (SR) model that replaces the deterministic reset of the potential after spike generation with a stochastic one. Let $t^{(f)}: u\left(t^{(f)}\right)=\vartheta$ be the firing time of a LIF neuron, then

$$
\lim _{t \rightarrow t^{(f)}, t>t^{(f)}} u(t)=\mathcal{N}\left(u_{r}, \sigma_{S R}\right)
$$


Stimulus | || || | | || || || | || || | || | | || | | || | | || ||| | | || | | || || || || || || ||||| || |
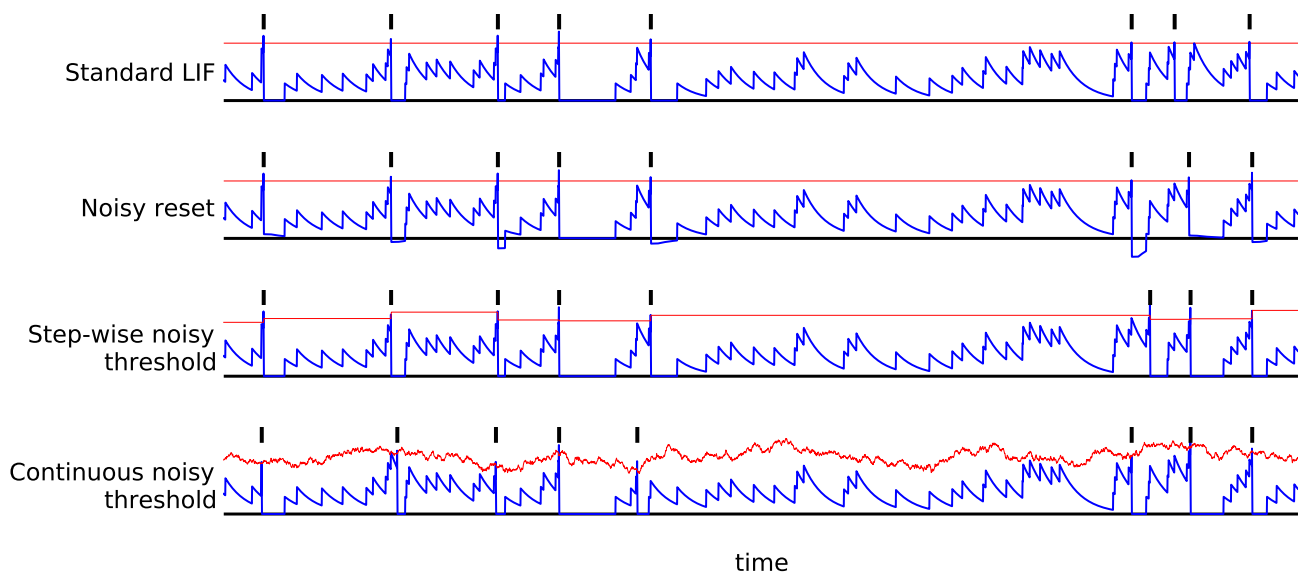

Figure 2: Evolution of the post-synaptic potential $u(t)$ and the firing threshold $\vartheta(t)$ over time (blue (dark) and yellow (light) curves respectively) recorded from a single neuron of each neural model. The input stimulus for each neuron is shown at the top of the diagram. The output spikes of each neuron are shown as thick vertical lines above the corresponding threshold curve.

defines the reset of the post-synaptic potential. $\mathcal{N}(\mu, \sigma)$ is a Gaussian distributed random variable with mean $\mu$ and standard deviation $\sigma$. Variable $\sigma_{S R}$ represents a parameter of the model.

We define two stochastic threshold models that replace the constant firing threshold $\vartheta$ of the LIF model with a stochastic one.

In the step-wise stochastic threshold (ST) model, the dynamics of the threshold update are defined as

$$
\lim _{t \rightarrow t^{(f)}, t>t^{(f)}} \vartheta(t)=\mathcal{N}\left(\vartheta_{0}, \sigma_{S T}\right)
$$

Variable $\sigma_{S T}$ represents the standard deviation of the Gaussian distribution $\mathcal{N}$ and is a parameter of the model. According to Eq. 3, the threshold is the outcome of a $\vartheta_{0^{-}}$ centered Gaussian random variable which is sampled whenever the neuron fires. We note that this model does not allow spontaneous spike activity. More specifically, the neuron can only spike at time $t^{(f)}$ when also receiving a pre-synaptic input spike at $t^{(f)}$. Without such a stimulus a spike output is not possible.

The continuous stochastic threshold (CT) model updates the threshold $\vartheta(t)$ continuously over time. Consequently, this model allows spontaneous spike activity, i.e. a neuron may spike at time $t^{(f)}$ even in the absence of a pre-synaptic input spike at $t^{(f)}$. The threshold is defined as an Ornstein-Uhlenbeck process [van Kampen, 2007]:

$$
\tau_{\vartheta} \frac{d \vartheta}{d t}=\vartheta_{0}-\vartheta(t)+\sigma_{C T} \sqrt{2 \tau_{\vartheta}} \xi(t)
$$

where the noise term $\xi$ corresponds to Gaussian white noise with zero mean and unit standard deviation. Variable $\sigma_{C T}$ represents the standard deviation of the fluctuations of $\vartheta(t)$ and is a parameter of the model. We note that $\vartheta(t)$ has an overall drift to a mean value $\vartheta_{0}$, i.e. $\vartheta(t)$ reverts to $\vartheta_{0}$ exponentially with rate $\tau_{\vartheta}$, the magnitude being in direct proportion to the distance $\vartheta_{0}-\vartheta(t)$.

The dynamics of the four models are presented in Figure 2. For each model a single neuron is shown that is stimulated by a random spike train generated by a Poisson process with mean rate $150 \mathrm{~Hz}$. Both the evolution of the post-synaptic potential $u(t)$ and the evolution of the firing threshold $\vartheta(t)$ are recorded and shown in the figure. We note the step-wise and the continuous update of the two threshold models and the stochastic reset of the reset model. Due to the stochastic dynamics each probabilistic model displays a different spike output pattern compared to the deterministic LIF neuron. 


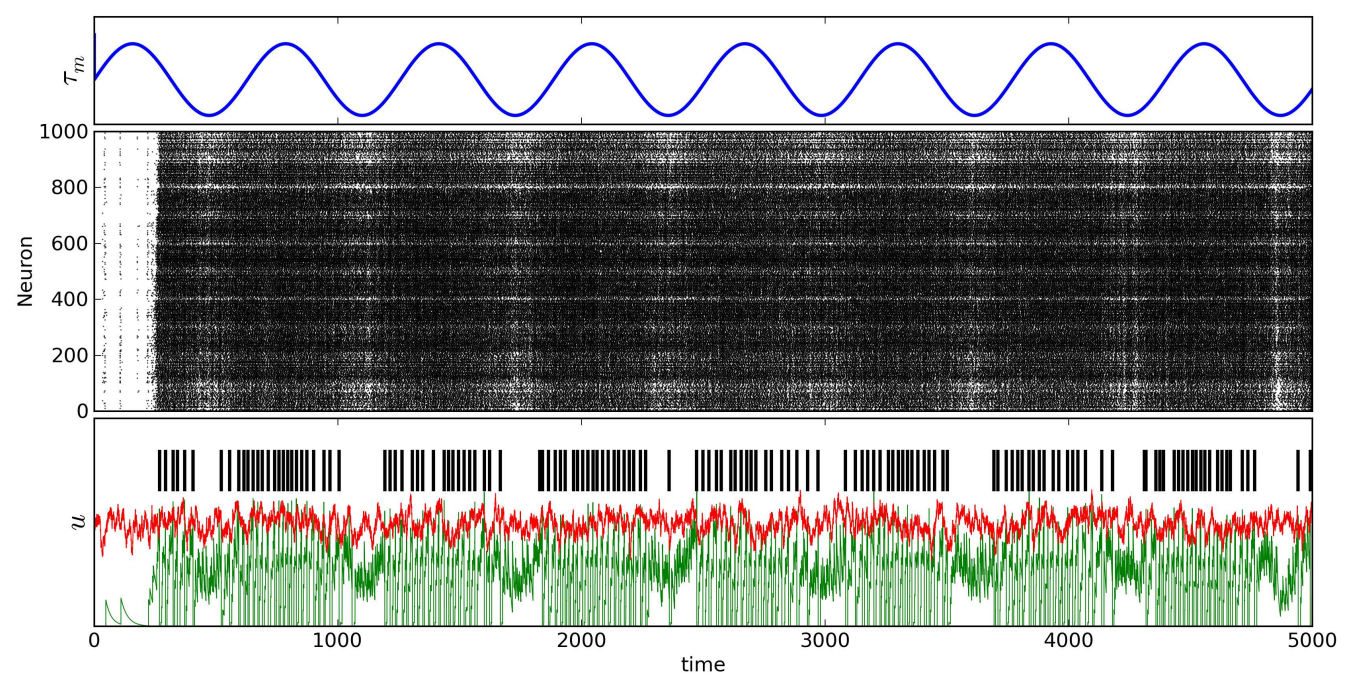

Figure 3: A Gene Regulatory Network (GRN) interacting with a spiking neural network (SNN) with 1000 neurons. The GRN controls a single parameter, i.e. the decay rate $\tau$, over a period of five seconds. The top diagram shows the evolution of $\tau$. The response of the SNN is shown as a raster plot of spike activity. A black point in this diagram indicates a spike of a specific neuron at a specific time in the simulation. The bottom diagram presents the evolution of the membrane potential of a single neuron in the network (green curve) along with its firing threshold $\vartheta$ (red curve). Spikes of the neuron are indicated as black vertical lines in the same diagram.

\section{Modelling the effect of gene dynamics on the spiking dynamics of a pSNN for a pCNGM}

Here we assume that a pSNN is built with the use of a probabilistic spiking neuron model and that the probability parameters are controlled by genes from the GRN. In order to illustrate the feasibility of modeling the effect of a gene that controls a probabilistic parameter on the functioning of the whole pSNN we have created the following experimental scenario. We constructed a reservoir having a small-world inter-connectivity pattern as described in [Maass et al., 2002]. A recurrent SNN is generated by aligning 1000 neurons in a three-dimensional grid of size $10 \times 10 \times 10$. In this grid, two neurons $A$ and $B$ are connected with a connection probability

$$
P(A, B)=C \times e^{\frac{-d(A, B)}{\lambda^{2}}}
$$

where $d(A, B)$ denotes the Euclidean distance between two neurons and $\lambda$ corresponds to the density of connections which was set to $\lambda=3$ in all simulations. Parameter $C$ depends on the type of the neurons. We discriminate into excitatory (ex) and inhibitory (inh) neural types resulting in the following parameters for $C: C_{e x-e x}=0.3, C_{e x-i n h}=$ $0.2, C_{i n h-e x}=0.4$ and $C_{i n h-i n h}=0.1$. The network contained $80 \%$ excitatory and $20 \%$ inhibitory neurons. All parameter values are directly adopted from [Grzyb et al., 2009].

The SNN is stimulated by a random spike train generated by a Poisson process with a mean rate of $75 \mathrm{~Hz}$. This stimulus is injected into 100 spiking neurons that were randomly selected from the reservoir.

The GRN is designed as a single gene that changes periodically its expression. It controls a single parameter of the SNN namely the decay rate $\tau_{m}$ of the LIF neuron. The value of $\tau_{m}$ is periodically modified ranging between values of 10 and $30 \mathrm{~ms}$. The impact of this temporal change is monitored in a computer simulation over five seconds of real time.

Figure 3 presents the results of the performed computer simulation. The top diagram shows the evolution of $\tau_{m}$ illustrating its periodic changes caused by the GRN output. The neural response of the SNN is depicted in the middle diagram. Here a point indicates the firing time of a particular neuron in the network. Clearly, the network is 
impacted by the change of its neural parameter $\tau_{m}$. Lower levels of $\tau_{m}$ result in a decreased activity of the spiking neurons. The same observation is made when looking at the evolution of the membrane potential of a single neuron in the network (lower diagram in Figure 3). The potential is represented by the green curve, while the red curve corresponds to the firing threshold $\vartheta$ of the neuron. We note the stochastic nature of the threshold. The output spikes of the neuron are shown as black vertical markers in the lower diagram. Clearly, the spike activity decreases for smaller values of $\tau_{m}$.

From this simple illustration, we conclude that the dynamics of the pSNN behaviour can be indeed controlled by the dynamics of a GRN as part of a pCNGM. By providing a quality measure for the behaviour of the pSNN, we can employ an optimization method, such as evolutionary algorithms, to match the pSNN output to a desired realworld dataset. After the optimization, a study of the GRN can lead to the discovery of new information.

\section{Conclusion and further research}

The paper demonstrated that using probabilistic models of spiking neurons in CNGM facilitates the study of the interaction between genes in the GRN model and the SNN behaviour. Further development of these models includes evolvability of the pSNN [Kasabov, 2007, Kasabov, 2009, Kasabov, 2008]. CNGM is a promising paradigm for future development of intelligent systems and their applications across disciplines. The models will potentially allow to model and trace the progress of neurodegenerative diseases under certain treatments and drugs [Kasabov et al., 2011]. Several pilot practical applications are aimed to be developed further across disciplines, including Bioinformatics, Neuroinformatics, Engineering, Economics and Social Sciences.

\section{Acknowledgments}

The work on this paper has been supported by the Knowledge Engineering and Discovery Research Institute (KEDRI, www.kedri.info). One of the authors, NK, has been supported by a Marie Curie International Incoming Fellowship within the $7^{\text {th }}$ European Framework Programme.

\section{References}

[Abbott, 1999] Abbott, L. F. (1999). Lapicque's introduction of the integrate-and-fire model neuron (1907). Brain Research Bulletin, 50(5-6).

[Benuskova and Kasabov, 2007] Benuskova, L. and Kasabov, N. (2007). Computational Neurogenetic Modelling. Springer, NY.

[Clopath et al., 2007] Clopath, C., Jolivet, R., Rauch, A., Lüscher, H.-R., and Gerstner, W. (2007). Predicting neuronal activity with simple models of the threshold type: Adaptive exponential integrateand-fire model with two compartments. Neurocomput., 70(10-12):1668-1673.

[Fogel, 2006] Fogel, D. B. (2006). Evolutionary computation - toward a new philosophy of machine intelligence (3. ed.). Wiley-VCH.

[Gerstner and Kistler, 2002] Gerstner, W. and Kistler, W. M. (2002). Spiking Neuron Models: Single Neurons, Populations, Plasticity. Cambridge University Press, Cambridge, MA.

[Grzyb et al., 2009] Grzyb, B. J., Chinellato, E., Wojcik, G. M., and Kaminski, W. A. (2009). Which model to use for the liquid state machine? In IJCNN'09: Proceedings of the 2009 international joint conference on Neural Networks, pages 1692-1698, Piscataway, NJ, USA. IEEE Press.

[Holter et al., 2001] Holter, J. L., Humphries, A., Crunelli, V., and Carter, D. A. (2001). Optimisation of methods for selecting candidate genes from cdna array screens: application to rat brain punches and pineal. Journal of Neuroscience Methods, 112(2):173 - 184.

[Kasabov, 2007] Kasabov, N. (2007). Evolving Connectionist Systems: The Knowledge Engineering Approach. Springer-Verlag New York, Inc., Secaucus, NJ, USA, second edition.

[Kasabov, 2008] Kasabov, N. (2008). Evolving intelligence in humans and machines: Integrative connectionist systems approach (feature article). IEEE Computational Intelligence Magazine, 3(3):23-37. 
[Kasabov, 2009] Kasabov, N. (2009). Integrative connectionist learning systems inspired by nature: current models, future trends and challenges. Natural Computing, 8:199-218. 10.1007/s11047-0089066-z.

[Kasabov, 2010] Kasabov, N. (2010). To spike or not to spike: A probabilistic spiking neuron model. Neural Networks, 23(1):16-19.

[Kasabov and Benuskova, 2004] Kasabov, N. and Benuskova, L. (2004). Computational neurogenetics. Journal of Computational and Theoretical Nanoscience, 1:47-61(15).

[Kasabov and Benuskova, 2005] Kasabov, N. and Benuskova, L. (2005). Theoretical and computational models for neuro, genetic, and neurogenetic information processing. In Rieth, M. and Schommers, W., editors, Handbook of Computational and Theoretical Nanotechnology, chapter 41. American Scientific Publishers, Los Angeles, CA.

[Kasabov et al., 2005] Kasabov, N., Benuskova, L., and Wysoski, S. G. (December 2005). Biologically plausible computational neurogenetic models: Modeling the interaction between genes, neurons and neural networks. Journal of Computational and Theoretical Nanoscience, 2:569-573(5).

[Kasabov et al., 2011] Kasabov, N., Schliebs, R., and Kojima, H. (2011). Probabilistic computational neurogenetic framework: From modelling cognitive systems to alzheimer's desease. IEEE Trans on Autonomous Mental Development. in print.

[Maass et al., 2002] Maass, W., Natschläger, T., and Markram, H. (2002). Real-time computing without stable states: A new framework for neural computation based on perturbations. Neural Computation, 14(11):2531-2560.

[Maass and Zador, 1999] Maass, W. and Zador, A. (1999). Dynamic stochastic synapses as computational units. In Advances in Neural Information Processing Systems, pages 903-917. MIT Press.

[Marcus, 2005] Marcus, G. F. (2005). The Birth Of The Mind: How A Tiny Number of Genes Creates the Complexities of Human Thought. Basic Books.

[Meng et al., 2010] Meng, Y., Jin, Y., Yin, J., and Conforth, M. (2010). Human activity detection using spiking neural networks regulated by a gene regulatory network. In Neural Networks (IJCNN), The 2010 International Joint Conference on, pages 1-6.

[Morse et al., 2010] Morse, A., de Greeff, J., Belpeame, T., and Cangelosi, A. (2010). Epigenetic robotics architecture (era). Autonomous Mental Development, IEEE Transactions on, 2(4):325 -339.

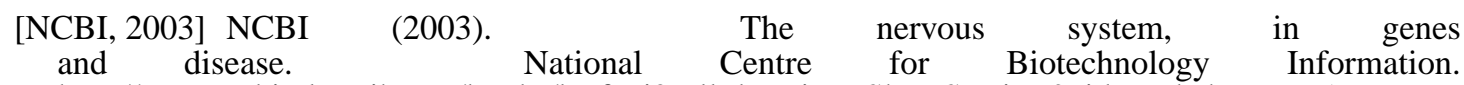
http://www.ncbi.nlm.nih.gov/books/bv.fcgi?call=bv.View..ShowSection\&rid=gnd.chapter.75.

[van Kampen, 2007] van Kampen, N. G. (2007). Stochastic Processes in Physics and Chemistry. NorthHolland.

[Villa et al., 2005] Villa, A. E. P., Asai, Y., Tetko, I. V., Pardo, B., Celio, M. R., and Schwaller, B. (2005). Cross-channel coupling of neuronal activity in parvalbumin-deficient mice susceptible to epileptic seizures. Epilepsia, 46(Suppl. 6):359. 